\title{
Adverse Changes in HbA1c, Body Weight and Insulin Use in People with Type 1 Diabetes Mellitus Following Dapagliflozin Discontinuation in the DEPICT Clinical Trial Programme
}

\author{
Jason Gordon • Thomas Danne - Lee Beresford-Hulme • \\ Hayley Bennet · Amarjeet Tank - Christopher Edmonds • \\ Fredrik Thorén · Markus Florian Scheerer · Phil McEwan
}

Received: February 19, 2020 / Published online: April 9, 2020

(C) The Author(s) 2020

\section{ABSTRACT}

Introduction: Dapagliflozin is an orally active inhibitor of sodium-glucose co-transporter 2 (SGLT2) that is indicated for use in adults with type 1 diabetes (T1DM) (with a body mass index (BMI) of at least $27 \mathrm{~kg} / \mathrm{m}^{2}$ in Europe, no such BMI limit in Japan), when insulin alone does not provide adequate glycaemic control. The

Enhanced Digital Features To view digital features for this article go to https://doi.org/10.6084/m9.figshare. 12005658.

Electronic supplementary material The online version of this article (https://doi.org/10.1007/s13300$020-00807-z)$ contains supplementary material, which is available to authorized users.

J. Gordon $(\varangle) \cdot$ L. Beresford-Hulme

Health Economics and Outcomes Research Ltd, Birmingham, UK

e-mail: jason.gordon@heor.co.uk

T. Danne

Hospital Auf der Bult, Hannover, Germany

H. Bennet · P. McEwan

Health Economics and Outcomes Research Ltd, Cardiff, UK

A. Tank - C. Edmonds

AstraZeneca, Cambridge, UK

F. Thorén

AstraZeneca, Göteborg, Sweden

M. F. Scheerer

AstraZeneca, Wedel, Germany aim of this study was to evaluate changes in glycated haemoglobin (HbA1c), body weight and insulin dose following discontinuation of dapagliflozin for the management of T1DM in the DEPICT clinical trial programme.

Methods: The interrelationship between treatment discontinuation, insulin requirement and outcomes post-discontinuation was evaluated using descriptive summary statistics and linear regression modelling. Data were analysed from individuals with T1DM discontinuing dapagliflozin in DEPICT-1 or DEPICT-2 (unplanned or end of study). HbA1c and body weight were measured over the 56-week study period (consisting of a 52-week treatment period and a 4-week follow-up period) at 4-8 weekly intervals. Following discontinuation of dapagliflozin, 1-year change in HbA1c (\%) and weight (kg) following discontinuation of dapagliflozin was estimated; total daily insulin doses were descriptively summarised.

Results: Of the 1059 individuals that received dapagliflozin during the DEPICT trials 91 met the eligibility criteria and were included in the analyses of HbA1c and body weight. The mean duration of follow-up was 209 days in both analyses. Following dapagliflozin discontinuation, estimated annualised changes in HbA1c and body weight were $+0.99 \%(95 \%$ CI $0.39,1.59)$ and $+3.75 \mathrm{~kg}(1.65,5.86)$, respectively. An increase in insulin dose was observed around the time of discontinuation; insulin dose in the 2 -week postdiscontinuation was $+3.6 \mathrm{IU}$ and $+4.4 \mathrm{IU}$ higher 
with dapagliflozin $5 \mathrm{mg}$ and $10 \mathrm{mg}$ than 2 weeks pre-discontinuation, respectively.

Conclusion: Discontinuation of dapagliflozin is predicted to lead to clinically meaningful increases in HbA1c and body weight, in addition to higher insulin doses. These findings are important in the management of people with T1DM among whom insulin is the only existing pharmacological treatment option.

Keywords: Body weight; Dapagliflozin; HbA1c; Treatment discontinuation; Type 1 diabetes mellitus

\section{Key Summary Points}

\section{Why carry out this study?}

The DEPICT clinical trial programme demonstrated that adding the sodiumglucose co-transporter 2 (SGLT2) inhibitor dapagliflozin to insulin therapy, in adults with type 1 diabetes mellitus (T1DM) and suboptimal glycaemic control, resulted in significant and clinically relevant reductions in $\mathrm{HbA1c}$, body weight and total daily insulin dose, compared to placebo.

As adherence to T1DM treatment can be a challenge, understanding the implications of treatment discontinuation on clinically relevant patient outcomes is important.

This study evaluated changes in HbA1c, body weight and insulin dose following discontinuation of dapagliflozin in the DEPICT trials.

\section{What was learned from the study?}

The study demonstrated that discontinuation of dapagliflozin is predicted to lead to clinically meaningful increases in HbA1c and body weight, in addition to higher insulin doses.

The results highlight the importance of ongoing treatment with dapagliflozin in the clinical management of T1DM to prevent the adverse changes associated with discontinuation.

\section{INTRODUCTION}

Type 1 diabetes mellitus (T1DM) is a chronic autoimmune disease, characterised by a progressive decline in pancreatic insulin production and a subsequent increase in glycated haemoglobin $(\mathrm{HbA1c})[1,2]$. The disease is most often diagnosed in children and adolescents, but it can also develop in adults in their late $30 \mathrm{~s}$ and early 40s [3].

Individuals with T1DM require lifelong insulin therapy consisting of daily multiple injections or continuous subcutaneous insulin infusions (via an insulin pump), with doses adjusted on the basis of frequent blood glucose self-monitoring or through closed-loop systems. A significant proportion of people have poor adherence to insulin treatment and therefore are unable to achieve or maintain HbA1c levels within the recommended limits [4]. It has been estimated that fewer than one in five people with T1DM are meeting HbA1c targets [5]. As intensive insulin therapy can result in an increased incidence of hypoglycaemia and substantial weight gain [6-8], the fear of these side effects is a barrier for many people with T1DM to correctly follow treatment advice [9]. For others the complexity of the treatment regimen i.e. the requirement of multiple daily insulin injections, testing blood glucose levels several times a day and adjusting treatment in relation to diet and exercise is a challenge [10]. Thus, there is a need for new therapies as an adjunct to insulin therapy, to better manage HbA1c and potentially lower the risk of onset of complications.

Dapagliflozin is a competitive, reversible, selective and orally active inhibitor of the human sodium-glucose co-transporter 2 (SGLT2), approved globally for people with type 2 diabetes mellitus (T2DM). It also has approval for use as an adjunct to insulin therapy for people with T1DM with a body mass index (BMI) of at least $27 \mathrm{~kg} / \mathrm{m}^{2}$, when insulin alone is unable to maintain glycaemic control, from the European Medicines Agency (EMA) and without the BMI restriction from Japan's Pharmaceuticals and Medical Devices Agency (PMDA) $[11,12]$. SGLT2 inhibition reduces glucose 
reabsorption in the renal tubule, leading to increased glucose excretion and consequently reducing hyperglycaemia and weight gain [13]. In addition to glycaemic effects, pleiotropic actions of SGLT2 inhibitors on cardio- and nephroprotection in T2DM have been demonstrated with several large trials [14-16]. There is the potential for such nephroprotection effects to be replicated in T1DM; however, studies to determine cardio-renal benefits in T1DM are currently outstanding.

The EMA approval for dapagliflozin in people with T1DM is based on phase 3 data from the DEPICT (Dapagliflozin Evaluation in Patients with Inadequately Controlled Type 1 Diabetes) clinical programme. The programme consists of two clinical trials, DEPICT-1 (ClinicalTrials.gov number NCT02268214) and DEPICT-2 (ClinicalTrials.gov number NCT02460978), in which individuals with T1DM inadequately controlled by insulin were randomised to receive add-on dapagliflozin (5 $\mathrm{mg}$ or $10 \mathrm{mg}$ ) or placebo orally, once daily for 52 weeks. The trial results demonstrated that dapagliflozin as adjunct therapy to insulin in individuals with inadequately controlled T1DM was well tolerated and there were significant and clinically relevant reductions in HbA1c, body weight and total daily insulin dose, compared to placebo, at both $5 \mathrm{mg}$ and $10 \mathrm{mg}$ doses [17-19]. Furthermore, in pooled analyses of 24-week continuous glucose monitoring data from DEPICT-1 and DEPICT-2, individuals receiving dapagliflozin $5 \mathrm{mg}$ or $10 \mathrm{mg}$ both spent more time in range compared to those receiving placebo [20], according to the international consensus on time in range recommendations of a blood glucose range of 70-170 mg/dL (3.9-10.0 mmol/L) [21]. An increased risk of diabetic ketoacidosis (DKA) was reported in individuals with T1DM receiving dapagliflozin [17-19]. Since the risk of DKA appeared to be less in those who were overweight or obese (BMI at least $27 \mathrm{~kg} / \mathrm{m}^{2}$ ) [22] the EMA indication for dapagliflozin is only in these individuals with T1DM.

As adherence to T1DM treatment can be a challenge, understanding the implications of treatment discontinuation on clinically relevant patient outcomes is important. This study evaluated changes in $\mathrm{HbA1c}$, body weight and insulin dose following discontinuation of dapagliflozin in the DEPICT trials.

\section{METHODS}

\section{Study Population}

In this retrospective analysis, data from individuals in DEPICT-1 and DEPICT-2 who received dapagliflozin ( $5 \mathrm{mg}$ or $10 \mathrm{mg}$ ) were evaluated for inclusion in the analysis dataset. Eligible individuals for analysis included those who discontinued dapagliflozin prematurely (i.e. unplanned, in accordance with protocol guidelines for study drug discontinuation) and as planned at the end of the study (i.e. completed scheduled treatment). Full details of the trial design and patient disposition in the DEPICT studies has been previously described [17-19].

The previously conducted studies in the DEPICT clinical trial programme were conducted in accordance with the Declaration of Helsinki and Good Clinical Practice Guidelines as defined by the International Council for Harmonisation. DEPICT-1 and DEPICT-2 were approved by the institutional review boards and independent ethics committees for all participating centres. All participants provided written informed consent. For Japanese patients at least 18 to less than 20 years old, informed consent was obtained from their parents/guardians.

\section{Endpoints and Data Structuring}

In this analysis, the primary endpoints of interest were $\mathrm{HbA1c}(\%)$ and body weight $(\mathrm{kg})$, with estimated total daily insulin dose (IU) dose considered as a secondary endpoint to provide context to the analysis of HbA1c and weight.

HbA1c and body weight were measured over the 56-week study period (consisting of a 52-week treatment period and a 4-week followup period) at 4-8 weekly intervals with additional assessments for body weight at weeks 1 and 2 post-baseline (study entry). Analysis of HbA1c and body weight considered individuals' 
post-discontinuation measurements to form HbA1c and weight trajectories following discontinuation of dapagliflozin. The analysis dataset for both HbA1c and weight trajectories consisted of all measurements post-discontinuation (last on- and all off-treatment), with individuals requiring at least three post-baseline measurements to evaluate trends (trajectories) in evaluated outcomes.

Total daily insulin doses were evaluated using insulin midpoint data (the mean of the highest and lowest dose received by the individual over the previous 2 -week period). Analyses considered insulin dose change from study baseline in absolute IU, as well as a percentage and included all recorded IU measurements. An index date for the analysis was defined as the fortnight in which discontinuation occurred. Fortnightly insulin midpoint data were then recoded relative to this index date; fortnightly periods before and after discontinuation were defined as weeks $-2,-4,-6$, etc. and 2, 4, 6, etc., respectively.

\section{Statistical Methods}

Baseline characteristics of individuals included in the analyses of post-discontinuation HbA1c and body weight trajectories were statistically summarised and compared to the overall population of dapagliflozin-treated individuals in the DEPICT studies. Reasons for dapagliflozin discontinuation were determined and mean HbA1c and body weight at discontinuation (last on-treatment observation) were calculated.

Ordinary linear regression models were used to estimate the time-dependent relationship between HbA1c and body weight following treatment discontinuation. Overall post-discontinuation trajectories of HbA1c and body weight were calculated as the mean of the individual patient trajectories, with an associated 95\% confidence interval (CI). Univariate summary statistics describe the mean number of observations and length of follow-up for individuals included in the analysis.

Insulin doses centred around the time of discontinuation were statistically summarised (mean and 95\% CI) for all individuals who received dapagliflozin to characterise the relationship between estimated HbA1c and body weight changes post-discontinuation and insulin doses.

\section{RESULTS}

\section{Study Population}

A total of 1059 individuals received dapagliflozin during the DEPICT trials and were potentially eligible for the analysis of post-discontinuation trajectories, of which 91 met the eligibility criteria and were included in the analyses of HbA1c and body weight. Owing to completeness of HbA1c and body weight data, the sample of included individuals differed slightly for each analysis; 89 individuals were included in both analyses. The baseline characteristics of those included in each analysis, as well as for all dapagliflozin-treated individuals in the DEPICT trials are summarised in Table 1.

Individuals included in the analyses were more likely to have been from DEPICT- 2 than DEPICT-1 (58.2\% versus $41.8 \%$ in both analyses). There was a similar proportion of individuals receiving a $10 \mathrm{mg}$ and $5 \mathrm{mg}$ dose of dapagliflozin in each analysis (52.7\% and 47.3\% in both analyses, respectively). The analysis populations were broadly similar to the pooled dapagliflozin-treated patient population of the DEPICT trials in terms of all other baseline characteristics. Mean HbA1c and weight at discontinuation for those included in each analysis was $8.21 \%$ and $79.00 \mathrm{~kg}$, respectively.

\section{Reasons for Dapagliflozin Discontinuation}

The most common reason for dapagliflozin discontinuation was adverse events [44/91 (48\%) and 43/91 (47\%) for the HbA1c and body weight analyses, respectively] (Table 2). The most frequent adverse events as reported by the investigator were metabolism and nutrition disorders (including ketosis, ketoacidosis and DKA) which were reported in 19 of 44 individuals who discontinued dapagliflozin because of adverse events in the HbA1c analysis and 19 of 
Table 1 Baseline characteristics

\begin{tabular}{|c|c|c|c|}
\hline \multirow[t]{2}{*}{ Baseline characteristic } & \multicolumn{2}{|c|}{ Individuals contributing to trajectory analysis } & \multirow[t]{2}{*}{ Full analysis set ${ }^{b}(N=1059)$} \\
\hline & HbA1c $(N=91)^{a}$ & Body weight $(N=91)^{\mathrm{a}}$ & \\
\hline \multicolumn{4}{|l|}{ Trial } \\
\hline DEPICT-1 & $38(41.8)$ & $38(41.8)$ & $518(48.9)$ \\
\hline DEPICT-2 & $53(58.2)$ & $53(58.2)$ & $541(51.1)$ \\
\hline \multicolumn{4}{|l|}{ Trial arm } \\
\hline Dapagliflozin $10 \mathrm{mg}$ & $48(52.7)$ & $48(52.7)$ & $529(50.0)$ \\
\hline Dapagliflozin $5 \mathrm{mg}$ & $43(47.3)$ & $43(47.3)$ & $530(50.0)$ \\
\hline \multicolumn{4}{|l|}{ Age (years) } \\
\hline$N \#$ & 91 & 91 & 1059 \\
\hline Mean (SD) & $40.77(13.35)$ & $40.82(13.52)$ & $42.46(13.57)$ \\
\hline Median $(\min , \max )$ & $43.00(18.00,70.00)$ & $43.00(18.00,70.00)$ & $43.00(18.00,75.00)$ \\
\hline \multicolumn{4}{|l|}{ Gender } \\
\hline Female & $53(58.2)$ & $53(58.2)$ & $579(54.7)$ \\
\hline Male & $38(41.8)$ & $38(41.8)$ & $480(45.3)$ \\
\hline \multicolumn{4}{|l|}{ Race (\%) } \\
\hline White & $83(91.2)$ & $83(91.2)$ & $924(87.3)$ \\
\hline Black or African-American & $4(4.4)$ & $5(5.5)$ & $23(2.2)$ \\
\hline Asian & $3(3.3)$ & $2(2.2)$ & $101(9.5)$ \\
\hline Other & $1(1.1)$ & $1(1.1)$ & $11(1.1)$ \\
\hline \multicolumn{4}{|l|}{ Body weight (kg) } \\
\hline$N \#$ & 91 & 91 & 1059 \\
\hline Mean (SD) & $81.60(20.71)$ & $81.37(20.68)$ & $80.44(17.89)$ \\
\hline Median (min, $\max )$ & $78.20(49.70,152.60)$ & $77.50(49.70,152.60)$ & $78.40(45.90,184.80)$ \\
\hline \multicolumn{4}{|l|}{$\operatorname{BMI}\left(\mathrm{kg} / \mathrm{m}^{2}\right)$} \\
\hline$N \#$ & 91 & 91 & 1059 \\
\hline Mean (SD) & $28.16(5.84)$ & $28.11(5.82)$ & $27.89(5.42)$ \\
\hline Median (min, $\max )$ & $26.70(18.60,43.90)$ & $26.70(18.60,43.90)$ & $27.20(18.20,65.80)$ \\
\hline \multicolumn{4}{|l|}{ Geographic region (\%) } \\
\hline Asia/Pacific & $5(5.5)$ & $3(3.3)$ & $120(11.3)$ \\
\hline Europe & $36(39.6)$ & $36(39.6)$ & $480(45.3)$ \\
\hline Latin America & $8(8.8)$ & $9(9.9)$ & $127(12.0)$ \\
\hline
\end{tabular}


Table 1 continued

Baseline characteristic
Individuals contributing to trajectory

analysis

HbA1c $(N=91)^{\mathrm{a}} \quad$ Body weight

$(N=91)^{\mathbf{a}}$

North America

Duration of T1DM (years)

$N \#$

Mean (SD)

Median (min, max)

$42(46.2)$

88

19.08 (10.63)

$17.81(2.76,49.74)$

$43(47.3)$

88

$19.36(10.60)$

$18.15(2.76,49.74)$

Full analysis set

$(N=1059)$

Total baseline insulin (IU/kg)

$N \#$

Mean (SD)

Median (min, max)

Total baseline insulin (IU)

N\#

Mean (SD)

Median (min, max)

HbA1c (percent)

N\#

Mean (SD)

Median (min, max)

Fasting plasma glucose $(\mathrm{mg} / \mathrm{dL})$

N\#

Mean (SD)

Median (min, max)

Fasting plasma glucose $(\mathrm{mmol} / \mathrm{L})$

$N \#$

Mean (SD)

Median (min, $\max$ )

Method of insulin administration

Continuous subcutaneous insulin infusion

Multiple daily injections
91

$0.72(0.24)$

$0.67(0.32,1.46)$

91

$59.42(26.92)$

$50.86(21.10,169.23)$

91

$8.50(0.69)$

$8.40(7.50,10.40)$

91

$185.01(76.21)$

187.00 (64.00, 402.00)

91

10.27 (4.23)

$10.38(3.55,22.31)$

39 (42.9)

$52(57.1)$
91

$0.73(0.24)$

$0.69(0.32,1.46)$

91

$59.99(26.83)$

$52.14(21.10,169.23)$

91

$8.51(0.69)$

$8.50(7.50,10.40)$

91

$186.56(75.85)$

$187.00(64.00,402.00)$

1059

$184.51(77.79)$

$176.00(24.00,492.00)$

$8.48(0.69)$

$8.30(7.50,10.90)$
$N \#$

$53(58.2)$
1059

10.24 (4.32)

$9.77(1.33,27.30)$

$375(35.4)$

$684(64.6)$ 
Table 1 continued

\begin{tabular}{llll}
\hline Baseline characteristic & \multicolumn{2}{l}{ Individuals contributing to trajectory analysis } & Full analysis set ${ }^{\mathbf{b}}(\boldsymbol{N}=\mathbf{1 0 5 9})$ \\
\cline { 2 - 4 } & \multicolumn{1}{l}{ HbA1c $(\boldsymbol{N}=\mathbf{9 1})^{\mathbf{a}}$} & Body weight $\left(\boldsymbol{N = 9 1 ) ^ { \mathbf { a } }}\right.$ & \\
\hline Use of CGM & $71(78.0)$ & $71(78.0)$ & $715(67.5)$ \\
No & $20(22.0)$ & $20(22.0)$ & $344(32.5)$ \\
Yes & 91 & 1059 \\
Systolic blood pressure (mmHg) & $120.59(13.95)$ & $121.88(14.34)$ \\
N\# & 91 & $119.00(95.00,158.00)$ & $121.00(84.00,176.00)$ \\
Mean (SD) & $120.62(14.28)$ &
\end{tabular}

$B M I$ body mass index, CGM continuous glucose monitoring, CI confidence interval, HbAIc glycated haemoglobin; $N \#$ number of individuals with complete baseline measurement, $S D$ standard deviation, TIDM type 1 diabetes mellitus

a 89 individuals were included in both analyses

b Based upon pooled data from the DEPICT-1 and DEPICT-2 studies

Table 2 Reasons for discontinuation

\begin{tabular}{|c|c|c|}
\hline \multirow[t]{2}{*}{$\begin{array}{l}\text { Reason for discontinuation } \\
N(\%)\end{array}$} & \multicolumn{2}{|c|}{$\begin{array}{l}\text { Individuals } \\
\text { contributing to } \\
\text { trajectory analysis }\end{array}$} \\
\hline & $\begin{array}{l}\text { HbA1c } \\
(N=91)\end{array}$ & $\begin{array}{l}\text { Body } \\
\text { weight } \\
(N=91)\end{array}$ \\
\hline Adverse event & $44(48.4)$ & $43(47.3)$ \\
\hline $\begin{array}{l}\text { Completed scheduled treatment } \\
\text { (short- and long-term) }\end{array}$ & $17(18.7)$ & $17(18.7)$ \\
\hline $\begin{array}{l}\text { Completed short-term period } \\
\text { without progressing to long- } \\
\text { term period }\end{array}$ & $3(3.3)$ & $3(3.3)$ \\
\hline Lack of efficacy & $1(1.1)$ & $1(1.1)$ \\
\hline Poor/non-compliance & $1(1.1)$ & $1(1.1)$ \\
\hline Pregnancy & $4(4.4)$ & $4(4.4)$ \\
\hline $\begin{array}{l}\text { Subject request to discontinue } \\
\text { study treatment }\end{array}$ & $11(12.1)$ & $11(12.1)$ \\
\hline $\begin{array}{l}\text { Subject no longer meets study } \\
\text { criteria }\end{array}$ & $1(1.1)$ & $1(1.1)$ \\
\hline Other & $9(9.9)$ & $10(11.0)$ \\
\hline
\end{tabular}

Short-term treatment period defined as up to week 24. Long-term treatment period defined as up to week 52
43 individuals in the body weight analysis, and infections which were reported in 15 of 44 individuals who discontinued dapagliflozin because of adverse events in the HbA1c analysis and 15 of 43 individuals in the body weight analysis. Less common adverse events resulting in discontinuation included gastrointestinal disorders, musculoskeletal and connective tissue disorders, nervous system disorders, and renal and urinary disease (Supplementary Table 1).

Other reasons for dapagliflozin discontinuation included individuals completed their scheduled treatment, completed short-term treatment period (up to week 24) without progressing to long-term treatment period (up to week 52), lack of efficacy, poor/non-compliance, pregnancy, subject request to discontinue study treatment and subject no longer meets study criteria (Table 2 ).

\section{Changes in HbA1c, Body Weight and Insulin Dose Following Dapagliflozin Discontinuation}

Estimated annual trajectories for $\mathrm{HbA1c}$ and body weight post-discontinuation are summarised in Fig. 1. The mean numbers of observations included in the trajectories for HbA1c 
A
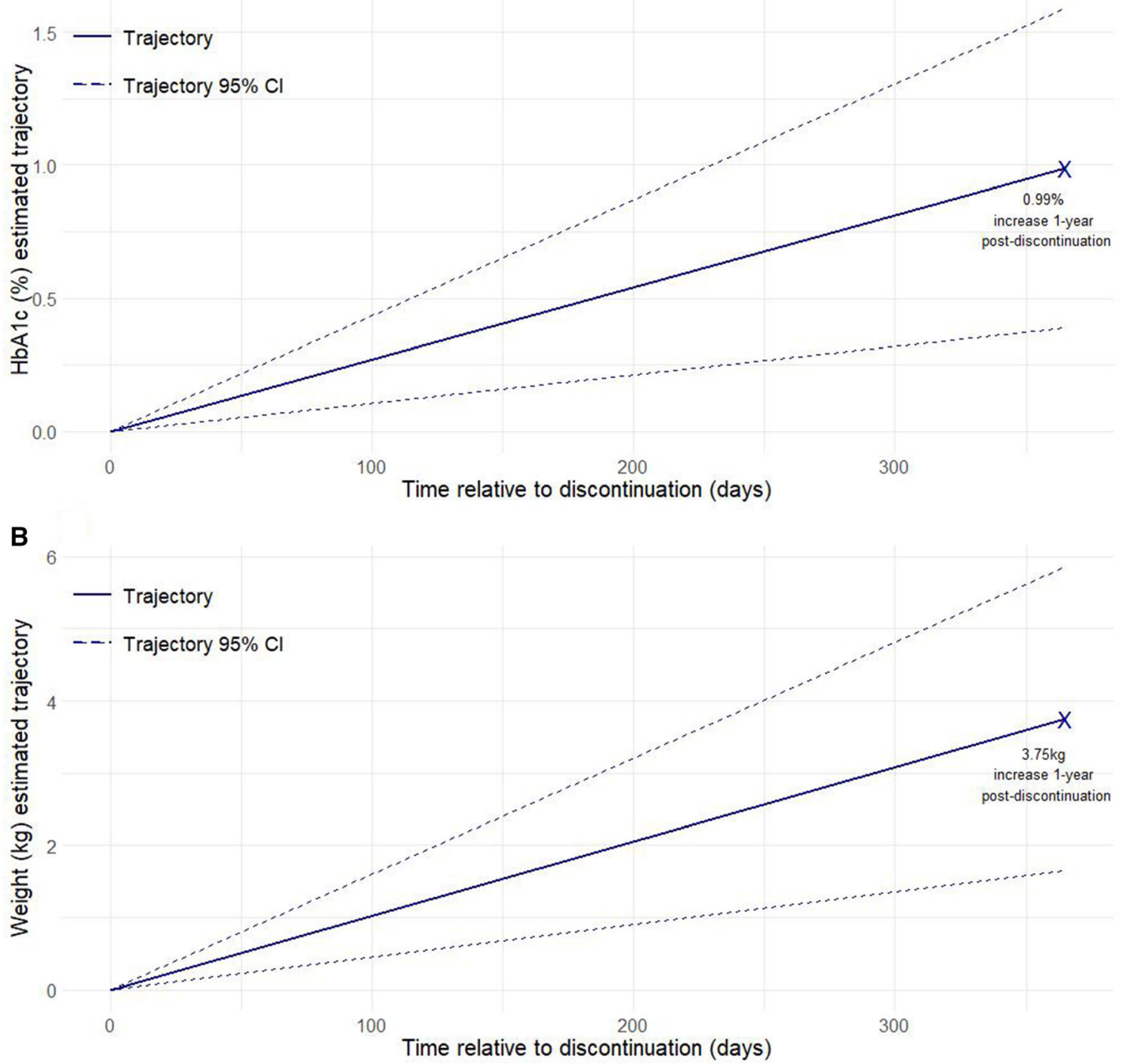

Fig. 1 Estimated trajectory of $\mathbf{a} \mathrm{HbAlc}$ and $\mathbf{b}$ body weight following discontinuation of dapagliflozin

and body weight were 5.5 (minimum 3 , maximum 10) and 5.6 (minimum 3, maximum 12) respectively, with a mean duration of follow-up of 209 (minimum 52, maximum 416) days in both analyses. The estimated mean increase in HbA1c was $0.99 \%$ per year (95\% CI 0.39-1.59). The estimated mean increase in body weight was $3.75 \mathrm{~kg}$ per year (95\% CI 1.65-5.86).

An increase in insulin dose was observed around the time of discontinuation when considering absolute change in IU and percentage change in IU from baseline (Fig. 2). Insulin dose
Fig. 2 a Insulin dose midpoint change from baseline relative to discontinuation. b Insulin dose midpoint percent change from baseline relative to discontinuation. Insulin midpoint values 16 weeks pre-discontinuation and 6 weeks post-discontinuation are included reflecting observed clustering of data for statistical evaluation (excluding outlier observations)

in the 2-week post-discontinuation time period was $+3.6 \mathrm{IU}$ and $+4.4 \mathrm{IU}$ higher in the dapagliflozin $5 \mathrm{mg}$ and $10 \mathrm{mg}$ arms than 2 weeks pre- 
A
A DAPA 5 MG +

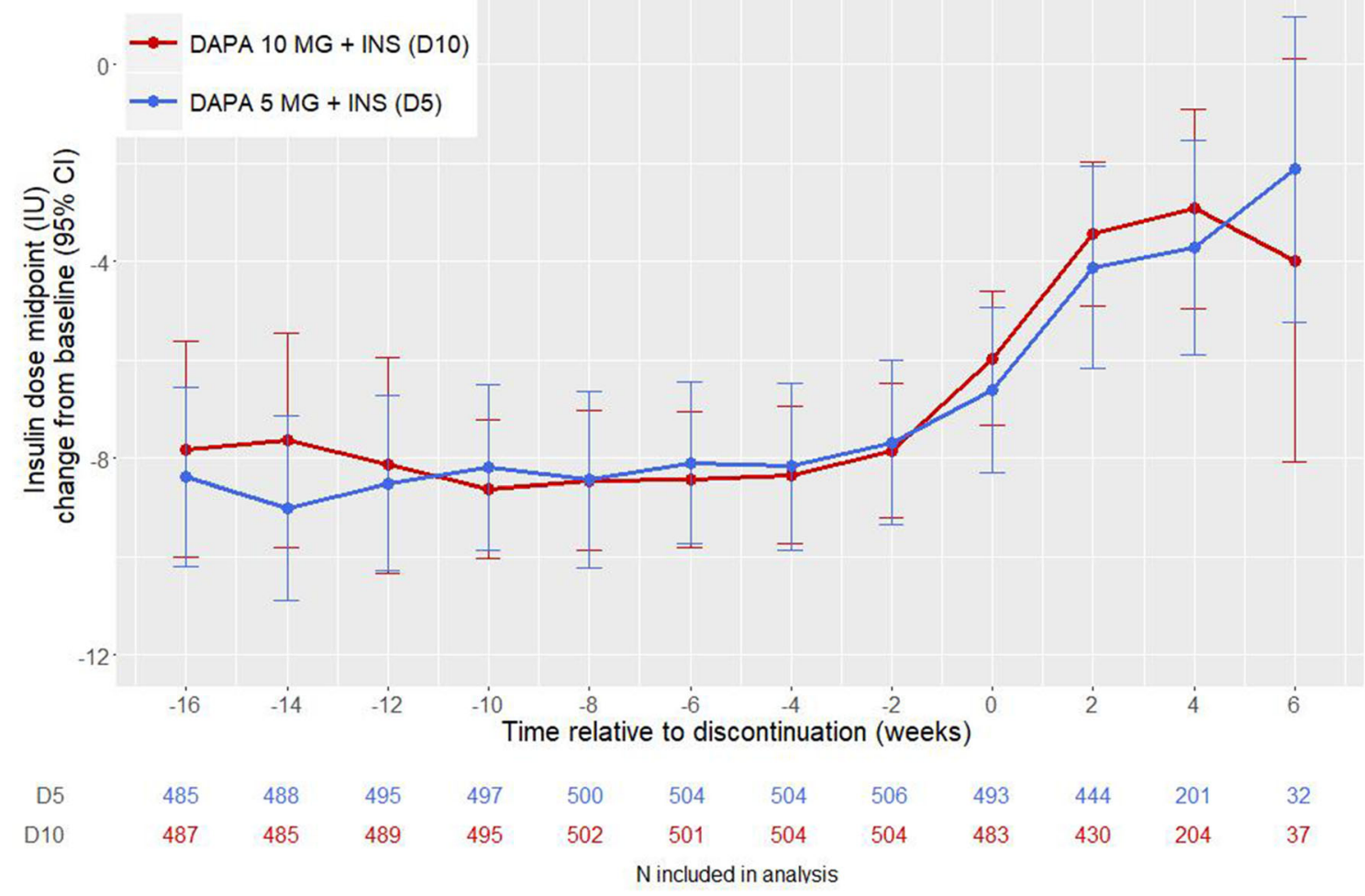

A
A DAPA 5 MG +

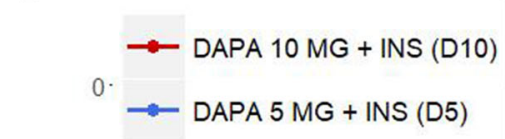

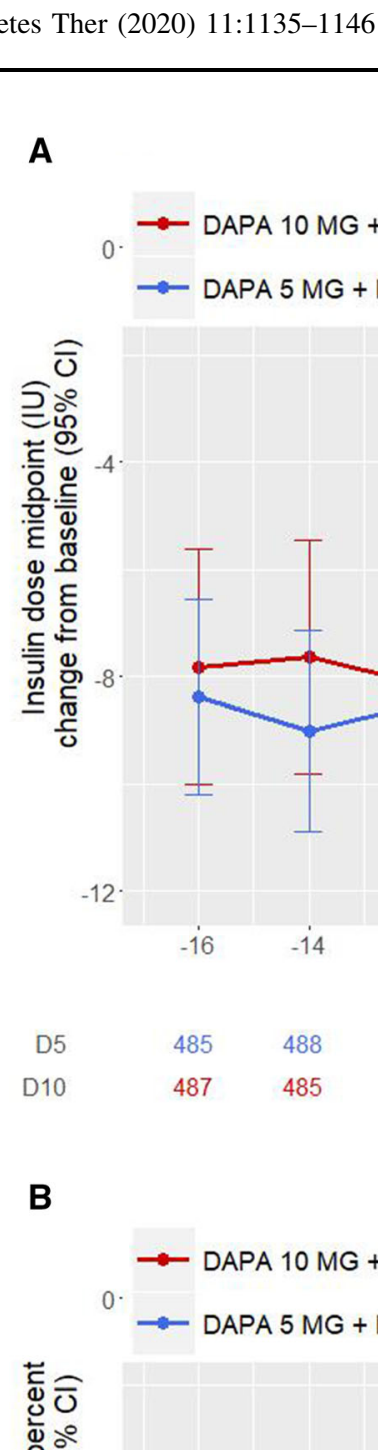

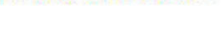


discontinuation, respectively. As a percentage change, insulin dose was $7.0 \%$ and $8.4 \%$ higher in the $5 \mathrm{mg}$ and $10 \mathrm{mg}$ arms, respectively. Insulin dose in the 4-week post-discontinuation time period was $+4.4 \mathrm{IU}$ and $+5.4 \mathrm{IU}$ higher in dapagliflozin $5 \mathrm{mg}$ and $10 \mathrm{mg}$ than 4 weeks prediscontinuation, respectively. As a percentage change, insulin dose in the 4 -week post-discontinuation time period was $7.8 \%$ and $8.1 \%$ higher, respectively.

\section{DISCUSSION}

Results of the DEPICT clinical trial programme have shown that treatment with dapagliflozin in individuals with T1DM is able to reduce both HbA1c levels and weight gain and requires few corrective insulin doses [17-19]. The results of this study indicate that HbA1c levels, body weight and insulin dose will increase following treatment discontinuation and suggest that, where clinically appropriate, ongoing treatment with dapagliflozin should be considered to prevent these changes associated with discontinuation. On the basis of American Diabetes Association (ADA) and National Institute for Health and Clinical Excellence (NICE) treatment guidelines, a change in HbA1c of $0.5 \%$ is considered clinically significant [23, 24]. Therefore, the estimated annual trajectories in HbA1c modelled in people with T1DM following discontinuation of dapagliflozin in this study (estimated mean annualised increase of $1 \%$ in $\mathrm{HbA1c}$ ) are of clinical importance.

The most common reason for early dapagliflozin discontinuation in this study was adverse events. As there are no progressive lines of therapy for T1DM, this study supports the importance of adverse event management, in order to avoid dapagliflozin discontinuation. Effective interventions to improve individuals' engagement in their long-term therapy, prevent treatment discontinuation and ultimately improve their clinical outcomes are an unmet need. This may include a range of strategies including educational resources, behavioural counselling or the use of digital technology to continually reinforce to individuals the importance of staying on treatment $[25,26]$.
This study is limited by the small sample size $(N=91$ for each analysis of time-dependent HbA1c and weight trajectories) as well as by the short-term nature of the follow-up of individuals following discontinuation: the maximum post-discontinuation follow-up for individuals was 56 weeks (i.e. the maximum follow-up in the DEPICT studies), with the majority of individuals having a shorter post-discontinuation follow-up. Therefore, inferences concerning long-term trajectories and their impacts should be treated with caution. Furthermore, the data utilised in this study reflects a clinical trial setting and therefore the results must be treated with caution when applying inferences to a realworld setting. Moreover, it is possible that the adverse changes in HbA1c, body weight and insulin use following dapagliflozin discontinuation may be a consequence of the return to 'real life' conditions out of the reinforced monitoring within a clinical study as well as physicians' and patients' inertia, rather than being as a result of the treatment discontinuation itself.

\section{CONCLUSION}

Discontinuation with dapagliflozin is predicted to be associated with statistically and clinically meaningful increases in HbA1c and body weight and is accompanied by increases in insulin doses. These findings are important in the management of people with T1DM among whom insulin is the only existing pharmacological treatment option.

\section{ACKNOWLEDGMENTS}

Funding. The DEPICT studies were sponsored by AstraZeneca and Bristol-Myers Squibb. This pooled post hoc analysis and the journal's rapid service fee were funded by AstraZeneca.

Medical Writing Assistance. Editorial assistance in the preparation of this article was provided by Dr Angharad R. Morgan of Health Economics and Outcomes Research Ltd. 
Support for this assistance was funded by AstraZeneca.

Authorship. All named authors meet the International Committee of Medical Journal Editors (ICMJE) criteria for authorship for this article, take responsibility for the integrity of the work as a whole, and have given their approval for this version to be published.

Disclosures. Thomas Danne has received speaker honoraria, research support and consulting fees from Abbott Diabetes Care, Bayer, BMS, AstraZeneca, Boehringer Ingelheim, Dexcom, Eli Lilly \& Company, Medtronic, Novo Nordisk, Sanofi, and Roche Diabetes Care and is a shareholder of DreaMed Diabetes. Amarjeet Tank, Christopher Edmonds and Fredrik Thorén are full-time employees of AstraZeneca. Markus Florian Scheerer has been an employee of AstraZeneca during the preparation of this manuscript and is currently employed at Bayer. Jason Gordon, Lee Beresford-Hulme, Hayley Bennet and Phil McEwan have received significant research grant funding from AstraZeneca in relation to this study.

Compliance with Ethics Guidelines. The previously conducted studies in the DEPICT clinical trial programme were conducted in accordance with the Declaration of Helsinki and Good Clinical Practice Guidelines as defined by the International Council for Harmonisation. DEPICT-1 and DEPICT-2 were approved by the institutional review boards and independent ethics committees for all participating centres. All participants provided written informed consent. For Japanese patients at least 18 to less than 20 years old, informed consent was obtained from their parents/guardians.

Data Availability. The datasets analysed during the current study were sourced from the original trial publications DEPICT-1 (ClinicalTrials.gov number NCT02268214) and DEPICT-2 (ClinicalTrials.gov number NCT02460978), the results of which are publicly available.
Open Access. This article is licensed under a Creative Commons Attribution-NonCommercial 4.0 International License, which permits any non-commercial use, sharing, adaptation, distribution and reproduction in any medium or format, as long as you give appropriate credit to the original author(s) and the source, provide a link to the Creative Commons licence, and indicate if changes were made. The images or other third party material in this article are included in the article's Creative Commons licence, unless indicated otherwise in a credit line to the material. If material is not included in the article's Creative Commons licence and your intended use is not permitted by statutory regulation or exceeds the permitted use, you will need to obtain permission directly from the copyright holder. To view a copy of this licence, visit http://creativecommons.org/licenses/by$\mathrm{nc} / 4.0 /$.

\section{REFERENCES}

1. Katsarou A, Gudbjornsdottir S, Rawshani A, et al. Type 1 diabetes mellitus. Nat Rev Dis Primers. 2017;3:17016. https://doi.org/10.1038/nrdp.2017. 16.

2. DiMzeglio LA, Evans-Molina C, Oram RA. Type 1 diabetes. Lancet. 2018;391(10138):2449-62. https://doi.org/10.1016/s0140-6736(18)31320-5.

3. MedScape. Type 1 diabetes mellitus 2019. http:// emedicine.medscape.com/article/117739-overview.

4. Peyrot M, Rubin RR, Kruger DF, Travis LB. Correlates of insulin injection omission. Diabetes Care. 2010;33(2):240-5. https://doi.org/10.2337/dc091348.

5. MedScape. Only $20 \%$ with type 1 diabetes are meeting HbA1c goals 2019. https://www.medscape. com/viewarticle/908314.

6. Nathan DM, Genuth S, Lachin J, et al. The effect of intensive treatment of diabetes on the development and progression of long-term complications in insulin-dependent diabetes mellitus. N Engl J Med. 1993;329(14):977-86. https://doi.org/10.1056/ nejm199309303291401.

7. The Diabetes Control and Complications Trial Research Group. Influence of intensive diabetes treatment on body weight and composition of 
adults with type 1 diabetes in the Diabetes Control and Complications Trial. Diabetes Care. 2001;24(10):1711-21.

8. Russell-Jones D, Khan R. Insulin-associated weight gain in diabetes-causes, effects and coping strategies. Diabetes Obes Metab. 2007;9(6):799-812. https://doi.org/10.1111/j.1463-1326.2006.00686.x.

9. Peyrot M, Skovlund SE, Landgraf R. Epidemiology and correlates of weight worry in the multinational Diabetes Attitudes, Wishes and Needs study. Curr Med Res Opin. 2009;25(8):1985-93. https://doi.org/ $10.1185 / 03007990903073654$.

10. Peyrot M, Barnett AH, Meneghini LF, SchummDraeger PM. Insulin adherence behaviours and barriers in the multinational Global Attitudes of Patients and Physicians in Insulin Therapy study. Diabetic Med. 2012;29(5):682-9. https://doi.org/10. 1111/j.1464-5491.2012.03605.x.

11. European Medicines Agency. Forxiga: EPAR - medicine overview. 2019. https://www.ema.europa.eu/ en/documents/overview/forxiga-epar-medicine-ov erview_en.pdf. Accessed 1 Oct 2019.

12. AstraZeneca K.K. and Ono Pharmaceutical Co Ltd. A selective sodium-glucose transporter (SGLT2)inhibitor Forxiga receives additional regulatory approval on indication and dosage and administration in type-1 diabetes in Japan. 2019. https:// www.ono.co.jp/eng/news/pdf/sm_cn190327_1.pdf. Accessed 1 Oct 2019.

13. Ferrannini E, Solini A. SGLT2 inhibition in diabetes mellitus: rationale and clinical prospects. Nat Rev Endocrinol. 2012;8(8):495-502. https://doi.org/10. 1038/nrendo.2011.243.

14. Zinman B, Wanner C, Lachin JM, et al. Empagliflozin, cardiovascular outcomes, and mortality in type 2 diabetes. N Engl J Med. 2015;373(22): 2117-28. https://doi.org/10.1056/nejmoa1504720.

15. Neal B, Perkovic V, Mahaffey KW, et al. Canagliflozin and cardiovascular and renal events in type 2 diabetes. N Engl J Med. 2017;377(7):644-57. https://doi.org/10.1056/nejmoa1611925.

16. Wiviott SD, Raz I, Bonaca MP, et al. Dapagliflozin and cardiovascular outcomes in type 2 diabetes. N Engl J Med. 2019;380(4):347-57. https://doi.org/ $10.1056 /$ nejmoa1812389.

17. Dandona P, Mathieu C, Phillip M, et al. Efficacy and safety of dapagliflozin in patients with inadequately controlled type 1 diabetes (DEPICT-1): 24 week results from a multicentre, double-blind, phase 3 , randomised controlled trial. Lancet Diabetes
Endocrinol. 2017;5(11):864-76. https://doi.org/10. 1016/s2213-8587(17)30308-X.

18. Dandona P, Mathieu C, Phillip M, et al. Efficacy and safety of dapagliflozin in patients with inadequately controlled type 1 diabetes: the DEPICT-1 52-week study. Diabetes Care. 2018;41(12):2552-9. https:// doi.org/10.2337/dc18-1087.

19. Mathieu C, Dandona P, Gillard P, et al. Efficacy and safety of dapagliflozin in patients with inadequately controlled type 1 diabetes (the DEPICT-2 Study): 24-week results from a randomized controlled trial. Diabetes Care. 2018;41(9):1938-46. https://doi.org/ 10.2337/dc18-0623.

20. Mathieu C, Dandona P, Phillip M, et al. Glucose variables in type 1 diabetes studies with dapagliflozin: pooled analysis of continuous glucose monitoring data From DEPICT-1 and -2. Diabetes Care. 2019;42(6):1081-7. https://doi.org/10.2337/ dc18-1983.

21. Battelino T, Danne T, Bergenstal RM, et al. Clinical targets for continuous glucose monitoring data interpretation: recommendations from the international consensus on time in range. Diabetes Care. 2019;42(8):1593-603. https://doi.org/10.2337/ dci19-0028.

22. Mathieu C, Dandona P, Phillip M, et al. SAT-LB025 Analysis of benefit/risk in the subgroup of patients with BMI of $>=27 \mathrm{~kg} / \mathrm{m}^{2}$ in the dapagliflozin DEPICT-1 and-2 trials in type 1 diabetes. J Endocr Soc. 2019;3(Supplement_1):25.

23. American Diabetes Association. Standards of medical care in diabetes-2009. Diabetes Care. 2009;32(Suppl 1):13-61. https://doi.org/10.2337/ dc09-s013.

24. National Institute for Health and Clinical Excellence. Type 2 diabetes: newer agents for blood glucose control in type 2 diabetes2009. https://www. eerstelijnsprotocollen.nl/dynmedia/8e 5c5d97d8fb2 bb9949e7f98b729fc7f. Accessed 1 Oct 2019.

25. Viana LV, Gomes MB, Zajdenverg L, Pavin EJ, Azevedo MJ. Interventions to improve patients' compliance with therapies aimed at lowering glycated hemoglobin (HbA1c) in type 1 diabetes: systematic review and meta-analyses of randomized controlled clinical trials of psychological, telecare, and educational interventions. Trials. 2016;17:94. https://doi. org/10.1186/s13063-016-1207-6.

26. Evans M, Hicks D, Patel D, Patel V, McEwan P, Dashora U. Optimising the benefits of SGLT2 inhibitors for type 1 diabetes. Diabetes Ther. 2019. https://doi.org/10.1007/s13300-019-00728-6. 